\title{
Recomendaciones nutricionales de la Asociación Colombiana de Nutrición Clínica para pacientes hospitalizados con infección por SARS-CoV-2
}

\author{
Nutritional recommendations of the Colombian Association of Clinical \\ Nutrition for hospitalized patients with SARS-CoV-2 infection \\ Recomendações nutricionais da Associação Colombiana de Nutrição Clínica para \\ pacientes hospitalizados com infecção por SARS-CoV-2
}

\author{
Charles Bermúdez ${ }^{1 *}$, Fernando Pereira², Diana Trejos-Gallego ${ }^{3}$, Angélica Pérez $z^{4}$, Milena Puentes ${ }^{5}$, \\ Lina María López ${ }^{6}$, Clara Plata García ${ }^{7}$ Esperanza Moncada Parada ${ }^{8}$, Martha Elena Muñoz Peláez ${ }^{9}$, \\ Justo Olaya Ramírez ${ }^{10}$, Mauricio Chona ${ }^{11}$, Andrés Becerra ${ }^{12}$, Diana Cárdenas ${ }^{13}$
}

Recibido: 22 de marzo de 2020. Aceptado para publicación: 24 de marzo de 2020

Publicado primero en línea el 30 de marzo 2020.

https://doi.org/10.35454/rncm.v3n1.066

\section{Resumen}

Introducción: la Organización Mundial de la Salud declaró la enfermedad COVID-19 causada por el virus SARS-CoV-2 como pandemia el 11 de marzo de 2020. En Colombia, se registró el primer caso el día 6 de marzo de 2020. Sin precedentes, esta pandemia presenta desafíos para los pacientes y los sistemas de salud en el mundo. El objetivo de este documento es presentar las recomendaciones de las intervenciones nutricionales para pacientes hospitalizados con infecciones por SARS-CoV-2, con especial énfasis en el paciente en estado crítico.

Método: se presenta una revisión y síntesis de la evidencia actual sobre intervenciones nutricionales en pacientes hospitalizados en estado crítico, síndrome de dificultad respiratoria aguda, adultos mayores y pacientes polimórbidos. Para ello, se consultaron las principales guías de la ACNC, ASPEN y ESPEN.

Resultados: todos los pacientes con COVID-19 se deben beneficiar del proceso de cuidado nutricional: detectar, nutrir y vigilar. A los pacientes con riesgo de peores desenlaces clínicos y mayor morbimortalidad (adultos mayores y pacientes polimórbidos) se debería realizar el tamizaje nutricional para identificar el riesgo de desnutrición. Se sugiere utilizar las herramientas validadas y recomenda-
\end{abstract}

\section{Summary}

Introduction: The World Health Organization declared the disease COVID-19 caused by the SARS-CoV- 2 virus as a pandemic on March 11, 2020. In Colombia, the first case was registered on March 6 , 2020. Unprecedented, this pandemic presents challenges for patients and healthcare systems worldwide. The objective of this document is to offer recommendations on nutrition interventions in hospitalized patients with SARS-CoV-2 infection with special emphasis on the critically ill patient.

Method: We review and summarize the current evidence on nutrition interventions in critically ill hospitalized patients, patients with acute respiratory distress syndrome, older adults, and polymorbid patients, included in guidelines by the Colombian Association of Clinical Nutrition (ACNC, for its acron$\mathrm{ym}$ in Spanish), American Society for Parenteral and Enteral Nutrition, and the European Society for Clinical Nutrition and Metabolism.

Results: All patients with COVID-19 should benefit from the nutrition care process: Screening, Therapy, and Monitoring Patients at risk of worse clinical outcomes and increased morbidity and mortality, such as older adults and polymorbid pa-

\section{Resumo}

Introdução: a Organização Mundial da Saúde declarou a doença COVID-19 causada pelo vírus SARS-CoV-2 como uma pandemia no dia 11 de março de 2020. Na Colômbia, o primeiro caso foi registado a 6 de março de 2020. Sem precedentes, essa pandemia apresenta desafios para pacientes e sistemas de saúde em todo o mundo. O objetivo deste documento é apresentar as recomendações de intervenções nutricionais para pacientes hospitalizados com infecção por SARS-CoV-2.

Método: é apresentada como uma síntese das evidências atuais sobre intervenções nutricionais em pacientes internados em estado crítico, síndrome do desconforto respiratório agudo (SDRA), idosos e pacientes polimórbidos. Para isso, foram consultados os principais guias do ACNC, ASPEN e ESPEN.

Resultados: todos os pacientes com COVID-19 devem beneficiar do processo de cuidado nutricional: detectar: nutrir e monitorizar. Pacientes em risco de piores resultados clínicos e aumento da morbimortalidade (idosos e pacientes polimórbidos) devem ser rastreados para identificar o risco de desnutrição. É recomendável usar os elementos validados e recomendados pelo ACNC. Recomendase que todos os pacientes críticos inter- 
das por la ACNC. Se recomienda que todo paciente en estado crítico hospitalizado en UCI por más de 48 horas sea considerado en riesgo nutricional.

Conclusión: reconociendo que el estado nutricional del paciente es un factor de riesgo modificable, y que la terapia nutricional reduce las complicaciones, los costos en salud y mejora la calidad de vida de los pacientes, la ACNC recomienda que la prevención, el diagnóstico y el tratamiento de la desnutrición se incluyan rutinariamente en el tratamiento de los pacientes con COVID-19.

Palabras clave: COVID-19, SARS-CoV-2, desnutrición, cuidado nutricional. tients, should be screened to identify risk of malnutrition. The use of tools validated and endorsed by the ACNC is recommended. Critically ill patients hospitalized in the intensive care unit for more than 48 hours must be considered at risk of malnutrition.

Conclusion: Based on the knowledge that nutritional status is a modifiable risk factor, and that nutritional therapy reduces complications, health costs and improves the quality of life of patients, the ACNC recommends that prevention, diagnosis and treatment of malnutrition should be routinely included in the treatment of patients with COVID-19.

Keywords: COVID-19; SARS-CoV-2; Malnutrition; Nutritional care. nados em UTI por mais de 48 horas sejam considerados de risco nutricional.

Conclusão: reconhecendo que o estado nutricional do paciente é um fator de risco modificável e que a terapia nutricional reduz complicações, custos de saúde e melhora a qualidade de vida dos pacientes, o ACNC recomenda essa prevenção, diagnóstico e tratamento. A desnutrição deve ser rotineiramente incluída no tratamento de pacientes com COVID-19.

Palavras-chave: COVID-19, SARS-CoV-2, desnutrição, cuidados nutricionais.
1 Departamento de Nutrición y Soporte Nutricional. Clínica la Colina y Clínica del Country. Bogotá, D.C., Colombia. Presidente ACNC 2017-2021.

2 Grupo de Soporte Nutricional, Clínica Las Américas, Medellín, Colombia.

3 Unidad de Cuidados Intensivos Hospital Santa Sofía Manizales. Facultad de Medicina Universidad de Manizales, Colombia.

4 Departamento de Nutrición, Clínica Coal. Bogotá, D.C., Colombia. Secretaria ejecutiva ACNC 2017-2021.

5 Liga Contra el Cáncer - Seccional Bogotá. Bogotá, D.C., Colombia. Tesorera ACNC 2017-2021.

6 Grupo de Soporte Metabólico y Nutricional. Clínica Universitaria
Colombia. Bogotá, D.C., Colombia. Vice-presidente ACNC 2017-2021.

7 Hospital San Ignacio, Pontificia Universidad Javeriana, Bogotá, D.C., Colombia.

8 Hospital Universitario Erasmo Meoz, Cúcuta, Colombia.

9 Unidad de Cuidados Intensivos, Hospital General de Medellín. Universidad de Antioquia, Medellín, Colombia.

10 Universidad Surcolombiana - Grupo de Soporte Nutricional, Hospital Hernando Moncaleano, Neiva, Colombia.

11 Grupo de Soporte Metabólico y Nutricional. Clínica Universitaria Colombia. Bogotá, D.C., Colombia.

12 Hospital San Vicente Fundación. Rionegro. Antioquia, Colombia.

13 Instituto de Investigación en Nutrición, Genética y Metabolismo, Facultad de Medicina, Universidad El Bosque, Bogotá, D.C., Colombia.

lidad son las personas inmunocomprometidas, es decir, adultos mayores y polimórbidos (dos o más patologías crónicas) y personas desnutridas en general ${ }^{(2)}$. Estos factores, sumados a la estancia en la UCI, se asocian comúnmente con un alto riesgo de desnutrición, lo que representa per se un factor de riesgo adicional y relevante para una mayor morbilidad y mortalidad en las enfermedades crónicas y agudas ${ }^{(3-5)}$. También es importante señalar que los pacientes con COVID-19 requieren estadías prolongadas en la UCI para la estabilización del síndrome de dificultad respiratoria aguda (SDRA), lo que puede causar o empeorar la desnutrición. Las consecuencias de la desnutrición se encuentran ampliamente descritas y se pueden resumir en inmunidad alterada, aumento de la mortalidad, de la estancia hospitalaria y en los costos directos e indirectos de la atención en salud ${ }^{(6,7)}$. En el caso particular de pacientes con SDRA se sabe que 
la malnutrición se asocia a una peor función muscular respiratoria ${ }^{(8)}$, mayor susceptibilidad a infecciones ${ }^{(9)} y$ mortalidad ${ }^{(10)}$. Cuando la infección por COVID-19 se acompaña de náuseas, vómito y diarrea, compromete el estado nutricional ${ }^{(11)}$.

En el caso de infecciones causadas por otros virus como el virus de la influenza, diversos estudios han mostrado que los factores asociados a mortalidad son el tipo del virus (OR 7,1), desnutrición (OR 25), infecciones adquiridas en el hospital (OR 12,2), insuficiencia respiratoria $(\mathrm{OR} 25,8)$ e infiltrados en la radiografía del tórax $(\mathrm{OR} 6)^{(12)}$. Esto se explica por el estado inflamatorio causado por las citoquinas proinflamatorias $y$ activadoras de la apoptosis y el aumento del catabolismo proteico lo que resulta en un desgaste agudo del músculo esquelético ${ }^{(13)}$. Las alteraciones metabólicas y el consecuente aumento del gasto energético relacionado con el mayor trabajo ventilatorio conllevan al aumento en los requerimientos nutricionales. Revisiones más detalladas de esta respuesta metabólica se han publicado de manera reciente $\mathrm{e}^{(5,14)}$.

La Asociación Colombiana de Nutrición Clínica (ACNC), desde hace varías décadas, ha tenido como objetivo promover la terapia nutricional óptima en el marco del cuidado nutricional hospitalario para hacer frente a la alta prevalencia de desnutrición hospitalaria en el país (cerca de $50 \%$ ). Al promover la investigación y la educación en nutrición clínica, la misión de la ACNC impacta no solo la sociedad académica y científica sino también el gobierno y el sistema de salud colombiano. Por lo tanto, reconociendo que el estado nutricional del paciente es un factor de riesgo modificable, y que la terapia nutricional reduce las complicaciones, los costos en salud y mejora la calidad de vida de los pacientes, la ACNC recomienda que la prevención, el diagnóstico y el tratamiento de la desnutrición se incluyan en forma rutinaria en el tratamiento de los pacientes con COVID-19.

Este documento presenta las recomendaciones para el cuidado nutricional de los pacientes adultos hospitaliza- dos con COVID-19, con especial énfasis en el paciente en estado crítico. Se fundamenta opinión de expertos y en la síntesis de la evidencia actual sobre intervenciones nutricionales en pacientes hospitalizados en estado crítico, síndrome de dificultad respiratoria aguda (SDRA), adultos mayores y pacientes polimórbidos. Para ello se consultaron las principales guías de la ACNC, la Sociedad Americana de Nutrición Enteral y Parenteral (ASPEN) y la Sociedad Europea de Nutrición Clínica y Metabolismo (ESPEN).

\section{EL MODELO DE CUIDADO NUTRICIONAL}

La Declaración de Cartagena sobre el derecho a la nutrición en los hospitales ${ }^{(4)}$, define en el Principio \# 2 el cuidado nutricional como un proceso (detectar, nutrir y vigilar). A través de este proceso se garantiza la alimentación en condiciones de dignidad de la persona enferma y en casos necesarios la terapia nutricional con fórmulas especializadas de acuerdo con la patología y condiciones particulares del paciente.

La atención nutricional del paciente hospitalizado debe centrarse en el siguiente modelo: detectar, nutrir y vigilar (Figura 1) ${ }^{(15)}$. En la Figura 2 se muestra la clasificación de los estados de la enfermedad COVID-19 y posibles objetivos terapéuticos, incluyendo la terapia nutricional.

\section{Recomendación}

Los pacientes con COVID-19 deben beneficiarse del proceso completo de cuidado nutricional con el objetivo de mejorar los desenlaces clínicos.

\section{DETECTAR}

\section{Tamizaje nutricional}

El tamizaje nutricional busca identificar los pacientes desnutridos o en riesgo de desnutrición. Las herramientas de tamizaje validadas permiten identificar los
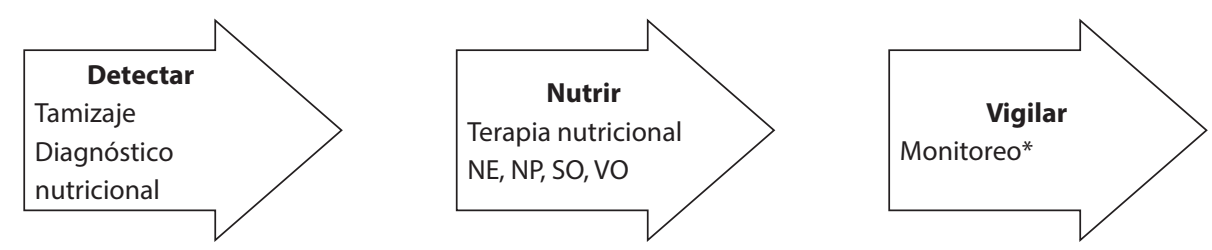

Figura 1. Etapas del cuidado nutricional según el Principio \# 2 de la Declaración de Cartagena. NE: nutrición enteral; NP: nutrición parenteral; SO: suplementos orales; VO: vía oral. * Monitoreo y seguimiento del plan nutricional intrahospitalario y plan de egreso hospitalario con manejo nutricional, que incluye la educación nutricional para el paciente, cuidador y familia. 
pacientes en riesgo nutricional y que se beneficiarían de la terapia nutricional. Se trata de herramientas estandarizadas, validadas, de fácil aplicación, y alta sensibilidad, con aceptación a nivel mundial (Tabla 1). Puede ser realizado por cualquier profesional de la salud y en las primeras $48 \mathrm{~h}$ del ingreso.

\section{Valoración nutricional}

La valoración nutricional debe ser realizada por profesionales en nutrición y dietética, dentro de las 24 horas siguientes a la generación de la interconsulta, en la cual se realizará el diagnóstico nutricional y se planteará una conducta nutricional acorde con las necesidades propias del paciente. Los criterios Global Leadership Initiative on Malnutrition (GLIM) permite, de acuerdo con criterios fenotípicos y genotípicos, hacer el diagnóstico y clasificar el estado nutricional ${ }^{(16)}$. Para el diagnóstico se requieren al menos un criterio fenotípico y uno etiológico (Tabla 2). La validez de estos pacientes no ha sido validada en los pacientes en UCI. La enfermedad de Covid-19, por su gravedad, debe considerarse como un criterio etiológico para el diagnóstico de desnutrición.

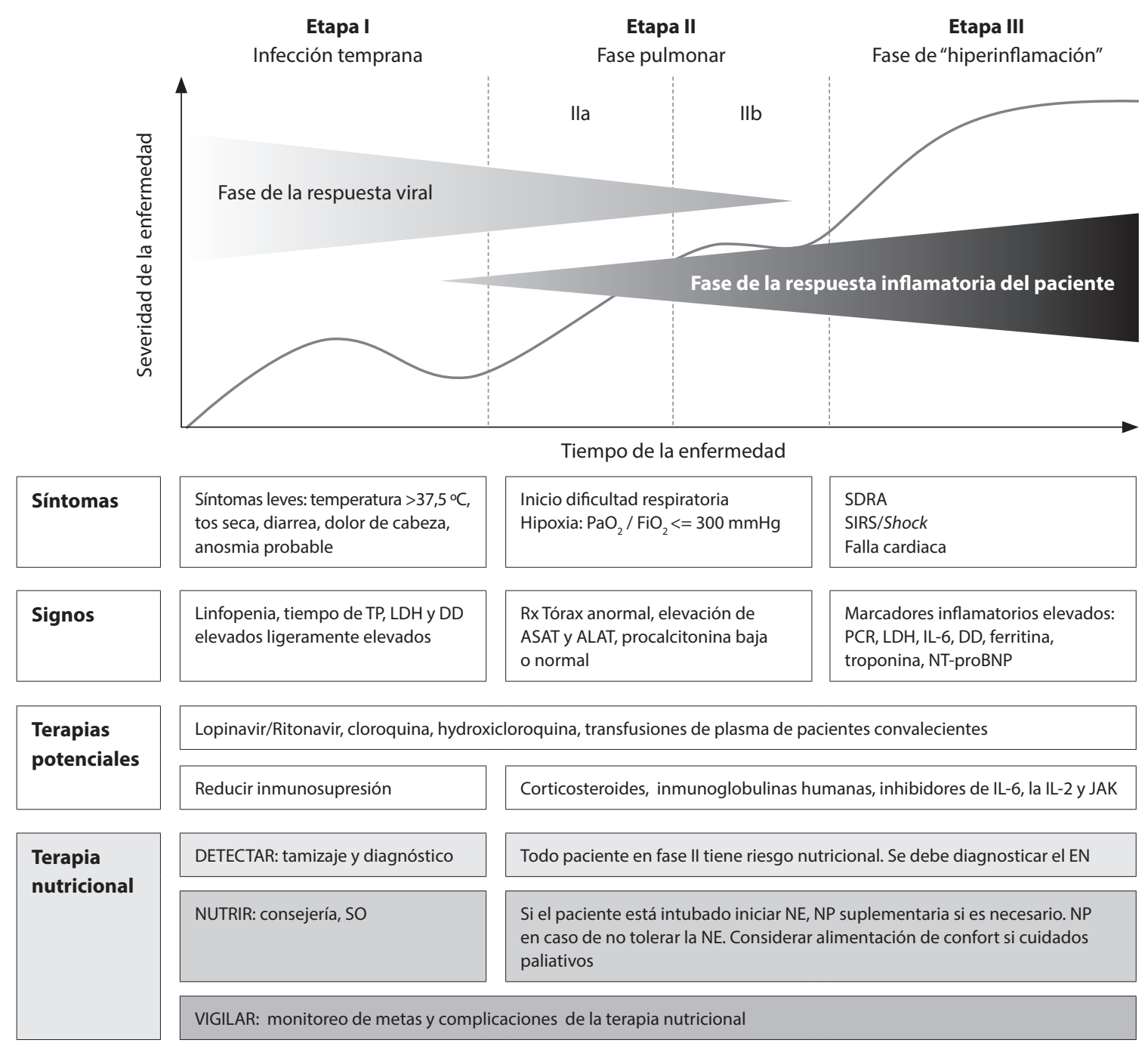

Figura 2. Clasificación de los estados de la enfermedad COVID-19 y posibles objetivos terapéuticos incluyendo la terapia nutricional. Se muestran 3 fases de progresión de la enfermedad con COVID-19, con signos asociados, síntomas y posibles terapias específicas. La terapia nutricional se debe intregrar en el tratamiento de la enfermedad. ALAT: alaninoaminotransferas; ASAT: aspartatoaminotransferasa; DD: D-dimeros; EN: estado nutricional; IL: interleucina; JAK: Janus quinasa; LDH: lactato deshidrogenasa; NE: nutrición enteral; NP: nutrición parenteral; PCR: proteína C reactiva; SDRA: síndrome de dificultad respiratoria aguda; SIRS: síndrome de respuesta inflamatoria sistémica; SO: suplementos nutricionales orales; TP: tiempo de protrombina. Adaptado de ${ }^{(30)}$. 
Tabla 1. Herramientas de tamizaje nutricional

\begin{tabular}{|l|l|}
\hline \multicolumn{1}{|c|}{ Población } & \multicolumn{1}{c|}{ Herramienta } \\
\hline Paciente adulto & Escala de Ferguson, NRS 2002 o MUST \\
\hline Paciente adulto mayor & MNA-SF \\
\hline Paciente en estado crítico & $\begin{array}{l}\text { Se recomienda considerar en riesgo nutricional a todo paciente en estado crítico con } \\
\text { más de 48 horas de estancia en UCl. }\end{array}$ \\
\hline
\end{tabular}

NRS 2002: Nutrition Risk Score, MUST: Malnutrition Universal Screening Tool, MNA-SF: MiniNutritional Assessment-Short Form.

Tabla 2. Criterios GLIM para el diagnóstico del estado nutricional en pacientes hospitalizados que no se encuentran en estado crítico $^{(16)}$.

\begin{tabular}{|l|l|l|l|}
\hline \multicolumn{2}{|c|}{ Criterio fenotípico } & \\
\hline Pérdida de peso $\%$ & $\begin{array}{l}>5 \% \text { en los pasados } 6 \text { meses o } \\
>10 \% \text { en más de } 6 \text { meses }\end{array}$ & $\begin{array}{l}\text { Ingesta de alimentos } \\
\text { reducida o disminución } \\
\text { en su asimilación }\end{array}$ & $\begin{array}{l}\text { Consumo de 50 \% de los requerimientos } \\
>1 \text { semana, o cualquier reducción por } \\
\text { más de 2 semanas, o cualquier afección } \\
\text { gastrointestinal que afecte la asimilación de } \\
\text { los alimentos }\end{array}$ \\
\hline $\begin{array}{l}\text { Bajo Índice de Masa } \\
\left.\text { Corporal (IMC } \mathrm{kg} / \mathrm{m}^{2}\right)\end{array}$ & $\begin{array}{l}<18,5 \mathrm{si}<70 \text { años o } \\
<22 \mathrm{si}>\text { años }\end{array}$ & Inflamación & $\begin{array}{l}\text { Enfermedad aguda/trauma o enfermedad } \\
\text { crónica* }\end{array}$ \\
\hline $\begin{array}{l}\text { Masa muscular } \\
\text { reducida }\end{array}$ & Medido por método confiable** & & \\
\hline
\end{tabular}

* La enfermedad de Covid-19, por su gravedad, debe considerarse como criterio etiológico para el diagnóstico de desnutrición.

** No se recomienda el uso de la dinamometría para evitar el contagio del virus entre los pacientes.

\section{Recomendación}

Los pacientes con COVID-19 con riesgo de peores desenlaces clínicos y una mayor morbimortalidad (adultos mayores y pacientes polimórbidos), deberán ser tamizados para identificar el riesgo de desnutrición. Utilizar las herramientas validadas y recomendadas por la ACNC (Tabla 1). Todo paciente en estado crítico hospitalizado en UCI por más de 48 horas debe ser considerado en riesgo nutricional.

\section{Recomendación}

Usar la estrategia GLIM para el diagnóstico nutricional de los pacientes que no estén hospitalizados en UCl. En los pacientes en estado crítico realizar una valoración general del estado nutricional que incluya anamnesis, valoración clínica, antropometría y marcadores bioquímicos.

\section{NUTRIR}

\section{Implementación de la terapia nutricional especializada}

La desnutrición hospitalaria es un proceso multicausal y de alta prevalencia, que requiere una intervención nutricional temprana con el fin de mejorar el pronóstico de los pacientes. La terapia nutricional incluye:

1. El abordaje terapéutico y consejería dietética para mejorar la calidad y cantidad de la alimentación

2. El uso de suplementos nutricionales orales ( $\mathrm{SO})$

3. La nutrición enteral (NE)

4. La nutrición parenteral (NP).

Dietoterapia y consejería nutricional: es la terapia nutricional encaminada a asegurar los requerimientos nutricionales durante enfermedades, mediante la ingesta de alimentos normales o enriquecidos de acuerdo con las condiciones particulares del paciente. Esto incluye modificaciones en el tamaño y consistencia de las porciones. La dietoterapia debe ser prescrita por profesionales en Nutrición y Dietética. Los pacientes con COVID - 19 pueden presentar deshidratación secundaria a la fiebre y a la dificultad respiratoria, que general mente viene acompañada de hiporexia. También pueden presentar vómito, náuseas y diarrea, lo que afecta la ingesta y absorción de alimentos ${ }^{(9)}$. Por tal razón es fundamental poder monitorizar por medio de contro- 
les de ingesta el consumo de alimentos e hidratación de tal manera que la primera estrategia debe ser la dietoterapia y la consejería nutricional (Tabla 3).

\section{Terapia nutricional oral}

La consejería nutricional busca alcanzar una alimentación saludable, es decir completa, equilibrada, suficiente y adecuada (CESA) además de variada e inocua. El protocolo para el equipo de salud que atenderá a pacientes con COVID-19 en el suministro de la alimentación debe regirse según la norma establecida en Colombia en la Resolución 2674 de 2013 del Invima y del Ministerio de salud.

\section{Recomendación}

Los pacientes en riesgo o en estado de desnutrición deberían optimizar su estado nutricional, idealmente a través de la consejería dietética realizada por un profesional idóneo.

Los pacientes hospitalizados con COVID-19 deben tener una alimentación saludable, cumpliendo con los requerimientos de calorías y nutrientes para el paciente adulto hospitalizado.

Mantener y vigilar la hidratación de los pacientes es importante. Se debe garantizar un aporte de 30 a $40 \mathrm{~mL} /$ $\mathrm{Kg}$ o 2,2 L / día para mujeres y $3 \mathrm{~L} /$ día para hombres ${ }^{(17)}$.

Los pacientes con COVID-19 también deben lograr la meta de consumir al menos 5 porciones de frutas $y$ verduras al día en el hospital.

El consumo de lácteos es indispensable en el marco de una alimentación equlibrada para la salud ósea en poblaciones con sedentarismo y postración en cama ${ }^{(18)}$. Los pacientes no deben suspender los productos lácteos y la leche, en algunos casos la leche deslactosada puede ser una elección.

En caso de que el paciente refiera síntomas que sugieran odinofagia, utilizar paletas de agua teniendo en cuenta la tolerancia a la glucosa de cada paciente. De ser posible la vía oral se sugiere iniciar dieta licuada espesa y revisar su consumo diario con escala visual de ingesta ${ }^{(19)}$.

Si la ingesta de dieta blanda o normal no cubre $100 \%$ del requerimento se sugiere iniciar suplementación nutricional $^{(19)}$.

Considerar la suplementación nutricional oral en pacientes con hospitalización en casa dado el riesgo nutricional después de la estancia en el hospital ${ }^{(18)}$.
Recomendación

Los pacientes con disfagia requieren que se modifique la textura de los alimentos. Algunos pueden requerir nutrición enteral por sonda. La terapia deglutoria deberá ser instaurada tan pronto se haga el diagnóstico de disfagia, y en todos los pacientes después de la extubación.

\section{Suplementación nutricional oral}

Se sugiere usar suplementación oral temprana (en las primeras 24 - 48 horas) cuando el paciente con el COVID-19 se encuentra desnutrido o en riesgo de desnutrición. Los pacientes en UCI o por fuera de ella deben ser tratados para prevenir el deterioro nutricional y mejorar el estado nutricional. La efectividad de la implementación de programas de suplementación nutricional tiene amplio respaldo científico. Más de 200 estudios clínicos sólidos, publicados en revistas indexadas y múltiples meta-análisis demuestran los efectos positivos de los suplementos en la disminución de complicaciones y mortalidad en los pacientes que lo requieren, $y$ en quienes no alcanzan a cubrir los requerimientos nutricionales con dieta por vía oral. Los suplementos nutricionales orales deben iniciarse cuando el paciente ingiere menos de $70 \%$ de los requerimientos nutricionales por vía oral, deben proveer $400 \mathrm{Kcal} /$ día, incluyendo al menos $30 \mathrm{~g} /$ día de proteína ${ }^{(20,21)}$.

\section{Recomendación}

Los suplementos nutricionales orales deben ser usados cuando el paciente no puede cubrir sus requerimientos nutricionales a pesar de una dieta adecuada y fortificada. Deben ser productos especialmente diseñados para este fin y prescritos de manera individual por profesionales con conocimientos en nutrición quienes determinarán la duración del tratamiento.

\section{Terapia nutricional enteral}

La no implementación de una terapia nutricional en pacientes desnutridos constituye un factor independiente y potencialmente modificable de peores resultados clínicos y mortalidad, por lo que identificar los pacientes con indicación de nutrición enteral por sonda es una prioridad, para instaurar una nutrición enteral de forma oportuna ${ }^{(22)}$. 


\section{Factores que favorecen la broncoaspiración}

a. Incapacidad de proteger las vías respiratorias

b. Ventilación mecánica

c. Edad $>70$ años

d. Nivel de conciencia reducido

e. Cuidado oral deficiente

f. Proporción inadecuada de enfermeras / pacientes

g. Posición supina

h. Déficit neurológico

i. Reflujo gastroesofágico

j. Transporte fuera de la UCI.

\section{Recomendaciones}

La terapia nutricional enteral debe ser indicada, prescrita y monitoreada por un equipo interdisciplinario de terapia nutricional. Debe ser la primera opción cuando se requiere terapia nutricional artificial siempre que el tubo digestivo esté funcionando.

En pacientes no intubados y que no alcanzan sus necesidades nutricionales con la dieta se deben usar suplementos nutricionales orales, en caso de no completar los requerimientos nutricionales con esta medida, se debe recurrir a la nutrición enteral.

En los pacientes en estado crítico intubados se debe iniciar nutrición enteral por una sonda nasogástrica temprana. Se recomienda la administración continua de la nutrición enteral. El uso de una sonda pospilórica se debe reservar para pacientes en decúbito prono, o en pacientes que no toleran la alimentación gástrica, y que no mejoran con proquinéticos (indicados de manera individual) o que están en alto riesgo de broncoaspiración. La pronación del paciente no es una contraindicación para la nutrición enteral.

\section{Terapia nutricional parenteral}

Hace parte fundamental del tratamiento nutricional de los pacientes que no pueden recibir alimentos por vía oral o enteral por sonda.

La terapia nutricional parenteral será indicada, prescrita y monitoreada por un equipo interdisciplinario de terapia médica nutricional para los pacientes con intolerancia parcial o total a la nutrición enteral o cuando esté contraindicada.

\section{Recomendación}

Los pacientes que no logren las metas energéticas y proteicas o que no toleran la vía enteral en la primera semana de intervención deben recibir nutrición parenteral. Esta no debe ser iniciada hasta agotar las estrategias para optimizar la tolerancia de la nutrición enteral.

\section{Requerimientos de vitaminas y micronutrientes}

El sistema inmunitario, complejo e integrado, necesita múltiples micronutrientes específicos, incluidas las vitaminas A, D, C, E, B6 y B12, ácido fólico, zinc, hierro, cobre y selenio, que desempeñan funciones vitales, a menudo sinérgicas en cada etapa de la respuesta inmune $^{(23,24)}$. El estado nutricional del huésped juega un papel muy importante en la defensa contra las enfermedades infecciosas.

Una nutrición inadecuada debilita la respuesta inmune, lo cual ha sido confirmado en estudios en humanos y en modelos experimentales ${ }^{(23,24)}$.

Las vitaminas $\mathrm{C}$ y $\mathrm{D}$ y el zinc son los micronutrientes con evidencia más fuerte de apoyo inmune. De acuerdo con estudios en otros virus, las deficiencias nutricionales afectan no solamente la respuesta inmune, sino también al patógeno viral. Es posible que las personas infectadas con el virus en esta pandemia tengan deficiencia de vitamina $\mathrm{D}$. En animales, la carencia de vitaminas $\mathrm{D}$ y E en terneros causó infección bovina con otros coronavirus ${ }^{(25)}$. En modelos celulares, la combinación de zinc y piritiona a bajas concentraciones inhibe la replicación del coronavirus del SARS (SARS-CoV) ${ }^{(22,23)}$.

\section{Recomendación}

Los pacientes en estado de desnutrición deben recibir, como parte de la terapia nutricional, los requerimientos individuales de vitaminas y minerales. La suplementación de vitaminas más allá de los requerimientos diarios no previene la infección por vírus ni han demostrado que mejore los desenlaces en los pacientes en estado crítico. En caso de carencias de micronutrientes se deben tratar de manera individualizada.

\section{Requerimientos nutricionales}

Los requerimientos de grasas y carbohidratos deben ser administrados teniendo en cuenta las necesidades energéticas, y considerando una relación de grasas : carbohidratos de 30:70 para pacientes que no estén en falla respiratoria. En caso de falla respiratoria y ventilación mecánica la proporción de carbohidratos debe disminuir; y por lo tanto, esta relación debe ser de 50:50.

En los pacientes en estado crítico, la energía no debe exceder $70 \%$ de los requerimientos e implementarse en los primeros 3 días de terapia, incrementando progresivamente a $100 \%$ después del día $3^{(3)}$. Se reco- 
mienda a los pacientes en estado crítico con COVID 19, la administración progresiva de 1,3 g / kg de proteínas por día ${ }^{(3)}$.

\section{USO DE PROBIÓTICOS Y PREBIÓTICOS EN PACIENTES CON COVID-19}

No existen evidencia de que los lácteos fermentados o los complementos de probióticos, prebióticos o simbióticos puedan ayudar, prevenir o disminuir el riesgo de infecciones en general. Sin embargo, algunos estudios identificaron disbiosis microbiana en varios pacientes con el COVID-19. Por lo tanto, es posible que el uso de prebióticos o probióticos (en forma de complementos alimenticios con variedad de cepas) pudiera evitar infección secundaria por translocación bacteriana $^{(18)}$; aunque la evidencia no es suficiente para recomendar su uso.

\section{Recomendaciones}

Calcular los requerimientos nutricionales teniendo en cuenta el estado del paciente (crítico o no crítico). No se recomienda el uso de calorimetría indirecta en este contexto epidémico donde se dificulta tener las precauciones higiénicas necesarias.

Se recomienda calcular el gasto energético a través de la medición de $\mathrm{VO}_{2}$ y $\mathrm{VCO}_{2}$ o de la ecuación basada en el peso: $25-30 \mathrm{kcal} / \mathrm{kg} /$ día.

El inicio de la nutrición debe ser lento y alcanzar la meta en 3 días por riesgo del síndrome de realimentación ${ }^{(17)}$.

Requerimiento proteico: administración progresiva de 1,3 g / kg de proteínas por día. Entre 1 y 1,5 g / kg en pacientes polimórbidos, ancianos. La meta es mantener la masa muscular del paciente.

\section{Recomendación}

Inicio temprano de rehabilitación física con el objetivo de mantener la masa muscular.

\section{NUTRICIÓN EN PACIENTES VENTILADOS QUE REQUIEREN PRONACIÓN}

Los pacientes tratados con ventilación mecánica en posición decúbito prono podrían tener un mayor riesgo de intolerancia a la nutrición enteral, debido a una serie de factores asociados a la alteración y disminución de la motilidad gástrica, retraso del vaciamiento gástrico, y a otras situaciones como la sedación con opiáceos y benzodiazepinas, altos valores de SAPS II y SOFA, sepsis, tratamiento con fármacos vasoactivos, hiperglucemia, posición supina sin elevación de la cabecera, y aumento de la presión intraabdominal, que pueden llevar a desarrollar signos y síntomas como vómito, broncoaspiración y neumonía asociada a la ventilación mecánica (NAV). Sin embargo, aunque la evidencia disponible es limitada y contradictoria, no parece ser el método más preciso para diagnosticar intolerancia a la nutrición enteral o para prevenir complicaciones, algunos protocolos incluyen el seguimiento de los volúmenes residuales gástricos (VRG) de manera intermitente $(<200 \mathrm{~mL}$ cada 3 a 6 horas, considerando VRG elevado $>500 \mathrm{~mL}$ ).

Como efecto preventivo, y con el objetivo de mejorar el vaciamiento gástrico y disminuir el riesgo de reflujo gastroesofágico y por consiguiente la neumonía asociada a ventilación, se deben tener en cuenta algunas estrategias incluyendo posición trendelemburg invertida con elevación de la cabecera 25 grados y el uso de medicamentos proquinéticos para aumentar la tolerancia.

Cuando no se logra cubrir los requerimientos por vía enteral, se deberá iniciar nutrición mixta con nutrición parenteral, para lograr llegar a las metas propuestas.

Los pacientes en estado crítico con hipoxemia severa, que se encuentran con ventilación mecánica invasiva y que requieren pronación, tienen mayor riesgo de desarrollar intolerancia a la nutrición enteral por lo expuesto anteriormente, pero se ha visto que es factible y seguro administrarla hasta alcanzar las metas propuestas, sin aumentar el riesgo de complicaciones gastrointestinales $^{(27,28)}$.

\section{NUTRICIÓN DE CONFORT O PALIATIVA EN PACIENTES CON NECESIDAD DE VENTILACIÓN MECÁNICA}

La pandemia de SARS-CoV-2 ha generado importantes dilemas éticos. Entre ellos la necesidad de elegir entre los pacientes en estado crítico que reciben ventilación mecánica y los que no debido a la falta de ventiladores. La ausencia de ventilación mecánica implica el empeoramiento de la neumonía y la muerte. Se trata de pacientes graves que con dificultad pueden alimentarse normalmente y cuya supervivencia a corto plazo es reducida. En estos casos, los cuidados deben dirigirse a mantener la mejor calidad de vida posible para el paciente. La nutrición artificial, enteral o parenteral no aportarían beneficio y sí podrían implicar efectos secundarios nefastos que alterarían su calidad de vida. Sin embargo, una alimentación de confort, indicada de manera individual puede ser iniciada. El paciente 
Tabla 3. Resumen de las recomendaciones de la ACNC sobre el cuidado nutricional de los pacientes hospitalizados con COVID-19

- La Asociación Colombiana de Nutrición Clínica recomienda que la prevención, el diagnóstico y el tratamiento de la desnutrición deben incluirse rutinariamente en el tratamiento de los pacientes con COVID-19.

- Los pacientes con el virus SARS-CoV-2 deben beneficiarse de un proceso completo de cuidado nutricional para mejorar los desenlaces clínicos y disminuir la mortalidad.

- Los pacientes con COVID-19 con riesgo de peores desenlaces clínicos y una mayor morbimortalidad (adultos mayores y pacientes polimórbidos), deberían ser tamizados para identificar el riesgo de desnutrición. Se sugiere utilizar las herramientas validadas y recomendadas por la ACNC.

- Todo paciente en estado crítico hospitalizado en UCI por más de 48 horas debería ser considerado en riesgo nutricional.

- Usar la estrategia GLIM para el diagnóstico nutricional de los pacientes que no estén hospitalizados en UCI. En los pacientes en estado crítico realizar una valoración general del estado nutricional que incluya anamnesis, valoración clínica antropometría y marcadores bioquímicos. Los pacientes desnutridos, con niveles importantes de inflamación, diabéticos, obesos y polimórbidos, requieren un abordaje nutricional diferenciado.

- Los pacientes en riesgo o en estado de desnutrición deberían optimizar su estado nutricional, idealmente por consejería dietética realizada por profesionales idóneos.

- Los pacientes con disfagia requieren que se modifique la textura de los alimentos. Algunos pueden requerir nutrición enteral por sonda. La terapia deglutoria deberá ser instaurada tan pronto se haga el diagnóstico de disfagia, y en todos los pacientes después de la extubación.

- Los pacientes tratados con ventilación mecánica en posición decúbito prono podrían tener un mayor riesgo de intolerancia a la nutrición enteral.

- Los suplementos nutricionales orales deben ser usados cuando el paciente no pueda cubrir sus requerimientos nutricionales a pesar de una dieta adecuada y fortificada. Deben ser productos especialmente diseñados para este fin y prescritos de manera individual por profesionales con conocimientos en nutrición quienes determinarán la duración del tratamiento.

- La terapia nutricional enteral debe ser indicada, prescrita y monitoreada por un equipo interdisciplinario de terapia nutricional. Debe ser la primera opción cuando se requiere terapia nutricional artificial siempre que el tubo digestivo se encuentre funcionando.

- En los pacientes no intubados y que no alcanzan a reunir sus necesidades nutricionales con la dieta, se deben usar suplementos nutricionales orales y si no se logra suplir con esta medida se debe recurrir a la nutrición enteral.

- En los pacientes en estado crítico intubados se debe iniciar nutrición enteral por una sonda nasogástrica. Se recomienda la administración continua de la nutrición enteral. El uso de una sonda pospilórica se debe reservar para pacientes en decúbito prono, o en pacientes que no toleran la alimentación gástrica, y que no mejoran con proquinéticos o que están en alto riesgo de broncoaspiración. La pronación del paciente no es una contraindicación para la nutrición enteral.

- Se debe iniciar con $70 \%$ de los requerimientos de energía en los primeros días, con incrementos hasta llegar a $100 \%$ en los siguientes tres días, de acuerdo con la evolución del paciente. La cantidad de proteína debe ser del orden de 1,3 g / kg / día, entre 1 y 1,5 g/ kg en pacientes polimórbidos, ancianos.

- Los pacientes que no logren las metas energéticas y proteicas o que no toleren la vía enteral en la primera semana de intervención deben recibir nutrición parenteral. Esta no debe ser iniciada hasta agotar las estrategias para optimizar la tolerancia de la nutrición enteral.

- Los pacientes en estado de desnutrición deben recibir, como parte de la terapia nutricional, los requerimientos individuales de vitaminas y minerales. La suplementación de vitaminas más allá de los requerimientos diarios no previene la infección por vírus ni han demostrado que mejore los desenlaces en los pacientes en estado crítico. En caso de carencias de micronutrientes se deben tratar de manera individualizada.

- Calcular los requerimientos nutricionales teniendo en cuenta el estado del paciente (crítico o no crítico/inflamados o no inflamados).

- Requerimiento de energía: 25-30 kcal / kg / día. Estos requerimientos deben ser ajustados de acuerdo con la condición de los pacientes. El inicio de la nutrición debe ser lento y alcanzar la meta en 3 días por riesgo del síndrome de realimentación.

- La cantidad de proteína debe ser del orden de 1,3 g/ kg / día. Entre 1 y 1,5 g / kg en pacientes polimórbidos, ancianos. La meta es mantener la masa muscular del paciente.

- Los requerimientos en grasas y carbohidratos deben ser administrados teniendo en cuenta las necesidades energéticas, y considerando una relación de grasa : carbohidrato de 30:70 para pacientes que no estén en falla respiratoria. En caso de falla respiratoria y ventilación mecánica la proporción de carbohidratos debe disminuir; y por lo tanto, esta relación debe ser de 50:50.

- En los pacientes en estado crítico, la energía no debe exceder $70 \%$ de los requerimientos e implementarse en los primeros 3 días de terapia, incrementan en forma progresiva a $100 \%$ después del tercer día. Realizar monitorización de la terapia nutricional y un plan de egreso hospitalario para asegurar la continuidad de un adecuado cuidado nutricional. Realizar rehabilitación física temprana, para el mantenimiento de la masa muscular, en todos los pacientes hospitalizados incluyendo atención en cuidado crítico.

- Los pacientes sometidos a cuarentena deben llevar una alimentación equilibrada y en lo posible, con la actividad física mientras se aseguran de tomar las precauciones necesarias para no contagiarse o propagar el virus.

- Los pacientes con COVID-19 que no son tratados con ventilación mecánica por falta de ventiladores deben beneficiarse de nutrición de confort en el marco de los cuidados paliativos. 
y su familia generalmente se sentirán más cómodos sabiendo que como parte de los cuidados paliativos se iniciará una alimentación de confort. La alimentación de confort tiene como objetivo proporcionar bienestar al comer para que el paciente se sienta cómodo física, social, emocional y espiritualmente. Apunta a la mejor calidad de vida posible y a aliviar los síntomas. Esto implica recomendar alimentos que el paciente puede tolerar, que representan una carga mínima y que corresponden a la forma en que el paciente maneja la progresión de la enfermedad ${ }^{(26)}$. Por lo tanto, ante esta situación la terapia nutricional enteral o parenteral se consideraría fútil, por lo que se sugiere una nutrición de confort, la cual acepta una ingesta nutricional insuficiente, puesto que su objetivo no es cumplir los requerimientos de nutrientes sino proporcionar bienestar $y$ la sensación de poder comer. Esto se logra por ejemplo humedeciendo los labios, hidratando y ofreciendo cantidades mínimas de alimentos de preferencia que pudieran ser soportados a pesar de la dificultad respiratoria $^{(29)}$.

\section{Recomendación}

Los pacientes con COVID-19 que no son tratados con ventilación mecánica por falta de ventiladores deben beneficiarse de nutrición de confort en el marco de los cuidados paliativos.

\section{MONITOREO Y SEGUIMIENTO DEL PLAN NUTRICIONAL}

Una terapia nutricional adecuada, suficiente y oportuna solo puede garantizarse cuando existe el monitoreo y seguimiento, pues de esta forma, se minimizan los riesgos y posibles complicaciones, además de optimizar los recursos.

En pacientes que requieren dietoterapia y suplementación nutricional oral, el monitoreo y seguimiento será realizado por un profesional en nutrición y dietética, quien será el encargado del registro en la historia clínica de las condiciones nutricionales del paciente, el logro de objetivos trazados, la indicación y forma de administración de la terapia, así como de las modificaciones y de su permanencia.

Para el caso de terapia nutricional enteral y parenteral, será el equipo interdisciplinario de terapia nutricional el encargado del registro diario del estado general, las condiciones metabólicas, la tolerancia y las modificaciones a la terapia que esta requiera.

\section{PLAN DE EGRESO HOSPITALARIO CON MANEJO NUTRICIONAL}

Los pacientes que fueron intervenidos, tendrán un plan de egreso individualizado que asegure la continuidad del tratamiento e incluyan al paciente en la toma de decisiones; todo lo anterior enfocado en garantizar un cuidado continuo y de esta forma obtener resultados en salud de mayor valor.

\section{Recomendaciones}

Realizar monitorización de la terapia nutricional y un plan de egreso hospitalario para asegurar la continuidad de un adecuado cuidado nutricional.

Los pacientes sometidos a cuarentena, aislamiento o distanciamiento social deben llevar una alimentación equilibrada y en lo posible, continuar con la actividad física mientras toman las precauciones necesarias para no contaminarse o propagar el virus.

\section{Financiación}

Estas recomendaciones no tuvieron financiación.

\section{Conflicto de intereses}

Los autores declaran no tener conflicto de interés.

\section{Declaración de autoría}

CH.B. realizó la búsqueda de la evidencia. CH.B. F.P. A.P., D.TG, M.CH., L.L., realizaron el análisis y las recomendaciones. Todos los autores escribieron, revisaron el artículo y validaron su versión final.

\section{Referencias bibliográficas}

1. Organizacion Mundial de la Salud. COVID 19. [Internet] (Consultado el 23 de marzo 2020). Disponible en: https:// www.who.int/emergencies/diseases/novel-coronavirus-2019/events-as-they-happen

2. Mehta PM, McAuley DF, Brown M, Sanchez E, Tattersall RS, Manson JJ. COVID-19: consider cytokine storm syndromes and immunosuppression. Lancet. 2020; published online March 16. https://doi.org/10.1016/S01406736(20)30628-0

3. Singer P, Blaser AR, Berger MM, Alhazzani W, Calder PC, Casaer MP, Hiesmayr M, Mayer K, Montejo JC, Pichard C, Preiser JC, van Zanten ARH, Oczkowski S, Szczeklik W, Bischoff SC. ESPEN guideline on clinical nutrition in the intensive care unit. Clin Nutr. 2019;38:48-79. 
4. Waitzberg D, Caiaffa WT, Correia MI. Hospital Malnutrition: The Brazilian National Survey (IBRANUTRI): A Study of 4000 Patients. Nutrition. 2001;17(7-8):573-80.

5. Correia MI, Waitzberg DL. The impact of malnutrition on morbidity, mortality, length of hospital stay and costs evaluated through a multivariate model analysis. Clin Nutr. 2003;22:235-9.

6. Jie B, Jiang ZM, Nolan MT, Efron DT, Zhu SN, Yu K, et al. Impact of nutritional support on clinical outcome in patients at nutritional risk: a multicenter, prospective cohort study in Baltimore and Beijing teaching hospitals. Nutrition. 2010;26:1088-93.

7. Giraldo Giraldo NA, Múnera García NE, Espitaleta Marrugo V, Pinerez LM. Prevalence of malnutrition and evaluation of dietary treatment for adult hospitalized patients in a public institution of high complexity. Perspect Nut Hum. 2007;9:37e47.

8. Pingleton SK, Harmon GS. Nutritional management in acute respiratory failure. JAMA. 1987;257(22):3094-9.

9. Rubinson L, Diette GB, Song X, Brower RG, Krishnan JA. Low caloric intake is associated with nosocomial bloodstream infections in patients in the medical intensive care unit. Crit Care Med. 2004;32(2):350-7.

10. Khalid I, Doshi P, DiGiovine B. Early enteral nutrition and outcomes of critically ill patients treated with vasopressors and mechanical ventilation. Am J Crit Care. 2010;19(3):261-8.

11. Chen N, Zhou M, Dong X, Qu J, Gong F, Han Y, Qiu Y, Wang J, Liu Y, Wei Y, Xia J, Yu T, Zhang X, Zhang L: Epidemiological and clinical characteristics of 99 cases of 2019 novel coronavirus pneumonia in Wuhan, China: a descriptive study. Lancet. 2020;395:507-513.

12. Reyes L, Arvelo W, Estevez A, Gray J, Moir JC, Gordillo B, et al. Population-based surveillance for 2009 pandemic influenza A (H1N1) virus in Guatemala, 2009. Influenza Other Respir Viruses. 2010;4:129-40.

13. Mervin Loi, Justin Wang, Chengsi Ong, Jan Hau Lee. Nutritional support of critically ill adults and children with acute respiratory distress syndrome: A clinical review. Clinical Nutrition ESPEN. 2017;19:1-8.

14. Preiser JC, Ichai C, Orban JC, Groeneveld AB. Metabolic response to the stress of critical illness. $\mathrm{Br} \mathrm{J}$ Anaesth. 2014;113(6):945-54.

15. Cárdenas D, Bermúdez $\mathrm{CH}$, Echeverri S, Pérez A, Puentes M, López M, et al. Declaración de Cartagena. Declaración Internacional sobre el Derecho al Cuidado Nutricional y la Lucha contra la Malnutrición. Nutr Hosp. 2019;36(4):97498. http://dx.doi.org/10.20960/nh.02701.

16. Cederholm T, Jensen GL, Correia MITD, Gonzalez MC, Fukushima R, Higashiguchi T, et al. GLIM criteria for the diagnosis of malnutrition - A consensus report from the global clinical nutrition community. Clin Nutr. 2019;38(1):1-9. doi: 10.1016/j.clnu.2018.08.002. Epub 2018 Sep 3.
17. Academia Española de Nutrición y Dietética, Consejo General de Colegios Oficiales de Dietistas-Nutricionistas. Recomendaciones de alimentación y nutrición para la población española ante la crisis sanitaria del COVID-19, marzo, 2020.

18. MinSalud, Recomendaciones de ingesta de energía y nutrientes RIEN para la población colombiana. 2016.

19. Pripp AH, Dahl OE. The population attributable risk of nutrition and lifestyle on hip fractures. Hip Int J Clin Exp Res Hip Pathol Ther. 2015;25(3):277-81.

20. Gomes F, Schuetz P, Bounoure L, Austin P, Ballesteros-Pomar M, Cederholm T, Fletcher J, Laviano A, Norman K, Poulia KA, Ravasco P, Schneider SM, Stanga Z, Weekes CE, Bischoff SC. ESPEN guideline on nutritional support for polymorbid internal medicine patients. Clin Nutr. 2018;37:336-353.

21. Volkert D, Beck AM, Cederholm T, Cruz-Jentoft A, Goisser S, Hooper L, Kiesswetter E, Maggio M, Raynaud-Simon A, Sieber CC, Sobotka L, van Asselt D, Wirth R, Bischoff SC. ESPEN guideline on clinical nutrition and hydration in geriatrics. Clin Nutr. 2019;38:10-4.

22. Bermúdez C, Sanabria A, Vega V. Soporte nutricional del paciente adulto, resultados de la reunión de consenso de la Asociación Colombiana de Nutrición Clínica. Rev Colomb Cir. 2013;28:101-116.

23. Gombart AF, et al. A Review of Micronutrients and the Immune System-Working in Harmony to Reduce the Risk of Infection. Nutrients. 2020;16:12(1). doi: 10.3390/ nu12010236

24. te Velthuis AJW, van den Worm SHE, et al. $\mathrm{Zn}(2+)$ inhibits coronavirus and arterivirus RNA polymerase activity in vitro and zinc ionophores block the replication of these viruses in cell culture. PLOS Pathog. 2010;6:e1001176. https://doi. org/10.1371/journal.ppat.1001176

25. Zhang L, Liu Y. Potential interventions for novel coronavirus in China: A systematic review. J Med Virol. 2020;92:479-90.

26. Beijer S, Jeanne Vogel, Harriet Jager-Wittenaar. Alternative terminology for the confusion term "palliative nutrition". Clin Nutr. 2017:36; 1723-4.

27. Reignier J, Dimet J, Martin-Lefevre L, Bontemps F, Fiancette $\mathrm{M}, \mathrm{Clementi} \mathrm{E}$ et al. Before-after study of a standardized ICU protocol for early enteral feeding in patients turned in the prone position. Clin Nutr. 2010;29(2):210-6. doi: 10.1016/j. clnu.2009.08.004

28. Saez de la Fuente I, Saez de la Fuente J, Quintana Estelles MD, Garcia Gigorro R, Terceros Almanza LJ, Sanchez Izquierdo JA, Montejo Gonzalez JC, et al. Enteral Nutrition in Patients Receiving Mechanical Ventilation in a Prone Position. JPEN J Parenter Enteral Nutr. 2016;40(2):250-5. doi: $10.1177 / 0148607114553232$.

29. Druml C, Ballmer PE, Druml W, Oehmichen F, Shenkin A, Singer P. et al. ESPEN guideline on ethical aspects of artificial nutrition and hydration. Clin Nutr. 2016;35(3):545-56. doi: 10.1016/j.clnu.2016.02.006. 
Bermúdez Ch, et al. Rev. Nutr. Clin. Metab. 2020;3(1):74-85.

30. Aiddiqi HK, Mehra MR. COVID-19 Illness in Native and Immunosuppressed States: A Clinical-Therapeutic Staging Proposal. Journal Of Heart And Lung Transplantation. [Internet] (consultado el 27 de marzo 2020). doi: 10.1016/j. healun.2020.03.012. Disponible en: MSchttps://els-jbsprod-cdn.literatumonline.com/pb/assets/raw/Health\%20 Advance/journals/healun/Article_2-1584647583070.pdf 